\title{
Ecología reproductiva de Pistacia lentiscus L. (Anacardiaceae): un anacronismo evolutivo en el matorral mediterráneo
}

\author{
Reproductive ecology of Pistacia lentiscus L. (Anacardiaceae): an evolutionary \\ anachronism in the mediterranean shrubland
}

MIGUEL VERDÚ \& PATRICIO GARCÍA-FAYOS

Centro de Investigaciones sobre Desertificación (CSIC-UV)

Apartado Oficial Albal, Valencia, E-46470, España; e-mail: Miguel.Verdu@uv.es

\begin{abstract}
RESUMEN
El lentisco (Pistacia lentiscus L., Anacardiaceae) es un arbusto esclerófilo de origen Oligocénico muy común en el Mediterráneo. Las condiciones climáticas imperantes en el momento en que se habría originado $P$. lentiscus eran de tipo tropical cálido, muy distintas a las mediterráneas actuales, por lo que muchos de los caracteres que presenta actualmente la especie pueden ser más un reflejo a las condiciones bajo las que evolucionó que a las condiciones actuales. Es en este sentido en el que se considera a esta especie como un anacronismo evolutivo. Para contestar a la pregunta de si el ser anacrónico significa poseer una estrategia inadecuada en las condiciones mediterráneas actuales, se ha realizado una revisión sobre la biología reproductiva de $P$. lentiscus. La conclusión principal es que el ciclo reproductivo de $P$. lentiscus está constituido por una amalgama de caracteres tropicales que le sirven no sólo para sobrevivir con éxito en el matorral mediterráneo, sino para colonizar los nuevos hábitats surgidos de la destrucción de éste.
\end{abstract}

Palabras clave: historia de vida, mediterráneo, Pistacia lentiscus, tropical.

\begin{abstract}
Pistacia lentiscus L. (Anacardiaceae) is a sclerophyllous shrub that is very common in the Mediterranean Basin. This species originated in the Oligocene under mild tropical conditions that were very different to the current mediterranean climate. For this reason, many of the plant characteristics should respond to the tropical -and not mediterraneanenvironmental pressures under it evolved. This is the fact why this species is considered to be an evolutionary anachronism. To answer the question if being anachronistic means to have a wrong strategy in the current Mediterranean conditions, a review of the reproductive biology of $P$. lentiscus has been carried out. The main conclusion is that the reproductive cycle of $P$. lentiscus is formed by a mixture of tropical characters that are useful not only to survive successfully in the Mediterranean shrublands but also to colonize new, disturbed, habitats.
\end{abstract}

Key words: life history, mediterranean, Pistacia lentiscus, tropical.

\section{INTRODUCCIÓN}

El clima tropical cálido reinante en la cuenca mediterránea durante el Terciario se fue modificando hasta conformar el actual clima mediterráneo, cuya antigüedad data de 3,2 millones de años (Raven 1973, Suc 1984). Suc (1984) basándose en datos paleoclimáticos y paleobotánicos propone una vegetación terciaria tipo laurisilva formada por helechos arborescentes, gimnospermas tipo Podocarpus y Sequoia y angiospermas perennifolias como Ilex, Ficus, Lindera, Laurus, Magnolia, Myrica, y palmeras como el género Sabal, que vivían en un clima de humedad y temperatura relativamente estable. La flora terciaria se fue extinguiendo, excepto ciertos taxa más xerófilos que empezaron a expandirse y diversificarse durante el Cuaternario desde los refugios xéricos en los que habían permanecido hasta entonces (Pons 1981). La extinción de la flora Terciaria parece que se ha producido aleatoriamente, ya que no ha sido identificada ninguna característica común entre los grupos extinguidos (Herrera 1992). Las especies xerófilas que se expandieron junto a las tropicales supervivientes y los representantes de la flora arctoterciaria de latitudes templadas que se añadieron a través de las migraciones producidas por las glaciaciones, conforman actualmente la flora mediterránea leñosa (Axelrod 1973, 1975).

En esta flora existe un claro gradiente morfológico-funcional en el que los extremos son identificados funcionalmente con las etapas de la sucesión y evolutivamente con su origen (Herrera 
1984b, 1992). Así, las especies de origen cuaternario se asocian a etapas primarias de la sucesión y las especies de origen Terciario lo hacen a etapas sucesionales más tardías. Las especies "cuaternarias" son típicamente caducifolias estivales facultativas, presentan hojas no esclerófilas ni coriáceas, flores grandes, hermafroditas y coloreadas, semillas pequeñas y polinización entomófila por insectos grandes. Las especies "tropicales" son típicamente perennifolias con hojas esclerófilas o coriáceas, flores reducidas, unisexuales y no coloreadas, semillas medianas o grandes, dispersión zoócora y polinización entomófila por insectos pequeños o anemófila.

Uno de los representantes típicos del grupo de especies que presentan afinidades tropicales es el lentisco ( $P$. lentiscus L., Anacardiaceae), un arbusto dioico de hasta $5 \mathrm{~m}$ de altura con hojas compuestas, perennes y esclerófilas distribuido en toda la cuenca mediterránea a bajas altitudes (Zohary 1952, Quézel 1981). Se han descrito fósiles del Oligoceno y Mioceno (Terciario) muy parecidos a los ejemplares actuales de Pistacia lentiscus (Pons 1981, Palamarev 1989). Dado que la tasa de evolución de P. lentiscus es, como el resto de las especies del género, muy lenta (Parfitt \& Badenes 1997), entonces muchos de los caracteres que presenta actualmente la especie serían más un reflejo de las condiciones bajo las que evolucionó que de las condiciones actuales. Es en este sentido en el que se considera a esta especie como un anacronismo evolutivo (Herrera 1992).

Pero, ¿significa el ser anacrónico poseer una estrategia inadecuada en las condiciones ambientales actuales? Para contestar esta pregunta y valorar hasta que punto $P$. lentiscus se desarrolla bien en el clima actual, se ha analizado la biología reproductiva de $P$. lentiscus a partir de la revisión de la información existente y se ha discutido a la luz de las condiciones actuales de las áreas donde habita.

\section{FLORACIÓN Y FRUCTIFICACIÓN}

La floración se produce entre marzo y abril solapándose la de ambos sexos (Jordano 1988, Correia et al. 1992, Martínez-Palle \& Aronne 2000, M. Verdú observaciones personales). Las flores masculinas tienen entre 8-10 estambres que producen $47-60 \times 10^{3}$ granos de polen por flor y están agrupados en inflorescencias de 8-10 flores (Jordano 1989). Los granos de polen son subcirculares o elípticos, con un diámetro mayor de 25-30 $\mu \mathrm{m}$ y un diámetro menor de 22-27 $\mu \mathrm{m}$
(Ballouche 1986). Las flores femeninas poseen un ovario tricarpelar y unilocular con un óvulo anátropo (Scaramuzzi 1957). Después de la polinización anemófila, los ovarios quedan latentes durante parte de la estación estival (Grundwag 1976). Posteriormente, mediante un crecimiento rápido, se desarrollan frutos carnosos con una única semilla. Los frutos son de color blanco y al madurar pasan por un color rojizo hasta alcanzar finalmente el negro. Los frutos de los tres colores coexisten en el mismo individuo y el cambio de tono se produce desde el mes de agosto hasta el de diciembre (De Lillis \& Fontanella 1992, M. Verdú observaciones personales). No todos los frutos terminan por madurar, ya que la maduración está fuertemente asociada a la viabilidad de la semilla. Así, los frutos negros son los que contienen la mayoría de las semillas viables y los rojos y blancos son partenocárpicos o presentan semillas abortadas (Jordano 1988, 1989).

El número de flores que finalmente forman frutos de tamaño final oscila en las distintas poblaciones estudiadas entre el 9 y el $53 \%$ (Tabla 1), habiendo una tendencia a incrementarse conforme aumenta la densidad poblacional (Verdú \& García-Fayos 1998a). Esto se explica porque en las poblaciones muy ralas no existiría suficiente polen para polinizar todas las flores y éstas caen. La limitación por polen desaparece a densidades por encima de 100 ind ha ${ }^{-1}$ (Verdú \& GarcíaFayos 1998a).

Sin embargo, muchos de estos frutos no poseen una semilla viable, de tal manera que se pueden encontrar tasas poblacionales de viabilidad de semillas que varían entre el 7 y $50 \%$ (Tabla 1 ). La viabilidad de las semillas de $P$. lentiscus no es dependiente de la densidad poblacional sino de los recursos hídricos. Así, individuos experimentalmente regados incrementaron significativamente el porcentaje de semillas viables al año siguiente del riego, mientras que los individuos no regados tuvieron la misma tasa de viabilidad ambos años (Verdú 1994).

En definitiva, las diferencias interindividuales en fructificación y crecimiento vegetativo están estrechamente asociadas a la historia pasada de cada individuo, como por ejemplo la acumulación de recursos, la depredación o la competencia. Por ello, las contingencias históricas son también responsables a escala microevolutiva de la supervivencia y reproducción de los individuos de esta especie.

También las contingencias históricas a nivel específico actúan sobre la fructificación. En este sentido, dos procesos limitantes en la fructificación como son el aborto y la partenocarpia tienen, además de una historia propia en cada individuo, 
una historia común en la especie. La partenocarpia aparece en cada individuo reproductor como un lastre filogenético con el que tiene que cargar por pertenecer a dicha especie.

\section{DEPREDACIÓN PREDISPERSIÓN DE LAS SEMILLAS}

Antes de ser dispersados, los frutos de P. lentiscus están sujetos a depredación por insectos (principalmente Megastigmus pistaciae, una avispa Calcidoidea) y vertebrados (principalmente aves Passeriformes). Mientras que los insectos ovipositan dentro de la flor, las aves depredan en el fruto. Las aves depredadoras de pulpa picotean el fruto dejando caer la semilla debajo de la planta madre sin dispersar y los depredadores de semilla separan la pulpa, abren la semilla e ingieren su contenido (Jordano 1989, 1990).

Los porcentajes de semillas dañadas por insectos suelen ser muy bajos, habiéndose descrito valores de 0,4-2,9\% de la cosecha total (Jordano 1989, Verdú \& García-Fayos 1998a). Los porcentajes de depredación por aves son ligeramente más altos, oscilando entre 3-31\% (Jordano 1989 , Verdú \& García-Fayos 1995, 2001).

Los frutos partenocárpicos y con semillas abortadas pueden disminuir la tasa de depredación por parte de las avispas, ya que las que ovipositan en estadíos florales tempranos son incapaces de detectar si la semilla desarrollará o no los tejidos necesarios para que la larva se alimente (Traveset 1993, Verdú \& García-Fayos 1998a). De la misma manera, la manipulación de frutos sin semilla o con semilla abortada conlleva, bajo ciertas condiciones, unos costos adicionales que pueden hacer a un pájaro granívoro no continuar depredando semillas en ese arbusto (Verdú \& GarcíaFayos 2001). Estos beneficios resultantes de engañar a los depredadores podrían explicar la paradoja de por qué los frutos partenocárpicos y con semillas abortadas son retenidos selectivamente en la planta. Se desconoce si Pistacia ha evolucionado recientemente este mecanismo de engaño a depredadores de frutos o ya lo poseía desde sus orígenes terciarios, pero lo que sí que se puede inferir a partir de un análisis filogenético es que la presencia de frutos partenocárpicos es un ca-

TABLA 1

Porcentaje de cuajado de frutos (número de frutos/flores) y de viabilidad de semillas (número de semillas viables/frutos) de P. lentiscus. Cuando una población fue estudiada más de un año, se indica el año tras un guión

Fruit set (number of fruits/flowers) and seed viability rates (number of filled seeds/fruits) from different populations of P. lentiscus

\begin{tabular}{lccl}
\hline Población & $\begin{array}{c}\text { Cuajado frutos } \\
(\%)\end{array}$ & $\begin{array}{c}\text { Semillas viables } \\
(\%)\end{array}$ & Referencia \\
\hline Columbretes (España) & 9,4 & 41,3 & $\begin{array}{l}\text { M. Verdú (resultados no publicados) } \\
\text { Martínez-Palle \& Aronne (2000) }\end{array}$ \\
Castelvolturno-97 (Italia) & 10 & & Herrera (1987) \\
Doñana (España) & 16,0 & & Verdú \& García-Fayos (1998a) \\
Petrer (España) & 17,9 & 9,4 & Martínez-Palle \& Aronne (2000) \\
Castelvolturno-96 (Italia) & 19 & 50 & Verdú \& García-Fayos (1998a) \\
Sagunto (España) & 19,4 & 9,8 & Martínez-Palle \& Aronne (2000) \\
Castelvolturno-90 (Italia) & 20 & & Abeltino (1996) \\
Rumanedda (Italia) & 21,3 & & Martínez-Palle \& Aronne (2000) \\
Castelvolturno-91 (Italia) & 21,8 & 44,5 & Jordano (1988) \\
Doñana-82 (España) & 26,8 & 6,9 & Verdú \& García-Fayos (1998a) \\
Duna Punta (España) & 30,9 & & Gulías \& Traveset (resultados no publicados) \\
Lluc (España) & 31,6 & & Gulías \& Traveset (resultados no publicados) \\
Lloret (España) & 39,3 & & Gulías \& Traveset (resultados no publicados) \\
Sa Bassa Plana (España) & 45,2 & & Gulías \& Traveset (resultados no publicados) \\
Esporles (España) & 47,8 & & Gulías \& Traveset (resultados no publicados) \\
Puigpunyent (España) & 50,3 & Verdú \& García-Fayos (1998a) \\
Porta-Coeli (España) & 51,6 & 11,7 & Verdú \& García-Fayos (1998a) \\
Pujol (España) & 52,9 & 19,9 & Jordano (1988) \\
Doñana-81 (España) & & 49 & Arista et al. (1990) \\
Grazalema (España) & & 20 & Grundwag (1975) \\
Jerusalem (Israel) & & &
\end{tabular}


rácter plesiomórfico (ancestral) no sólo del género Pistacia sino de su familia Anacardiaceae (Verdú \& García-Fayos 1998a). Por tal motivo, e independientemente del papel actual frente a los depredadores, es más parsimonioso pensar que la partenocarpia y el aborto son restricciones del desarrollo heredadas por P. lentiscus.

\section{DISPERSIÓN}

Un proceso anacrónico aunque efectivo del ciclo reproductor de $P$. lentiscus, y en general de las especies de origen terciario, es el de la dispersión zoócora. El proceso de extinción y reemplazamiento de la avifauna en la cuenca Mediterránea es similar a la sufrida por la vegetación. Los frugívoros que dispersaban las semillas durante el período Terciario sufrieron una extinción en Europa y un reemplazamiento por Passeriformes euroasiáticos que se diversificaron a finales del Terciario (Jordano 1984). No obstante, el reemplazamiento taxonómico de frugívoros no debió afectar a las especies cuyos dispersantes fueron sustituidos por otros de tipo generalista, como es el caso de P. lentiscus. En esta especie, los frutos son dispersados principalmente por aves Passeriformes durante el período de septiembre a marzo (Herrera 1984a, Jordano 1989, Verdú \& García-Fayos 1994). Las aves dispersantes de semilla ingieren el fruto entero, digieren la pulpa y regurgitan o defecan la semilla entera sin dañarla (Jordano 1984).

Los frutos de P. lentiscus son fuertemente preferidos por los frugívoros con respecto a los frutos de otras especies ya que tanto por su diseño como por su composición química poseen un altísimo índice de aprovechamiento (Herrera 1982a, 1988). El componente químico tan apreciado por los frugívoros sería la gran cantidad de lípidos que posee la pulpa, mientras que el diseño tan apropiado consiste en una gran cantidad de materia seca por unidad de peso del fruto ingerido (Herrera 1982a, 1982b, 1987, Debussche et al. 1987). Estas características hacen que el éxito de dispersión sea de intermedio a muy alto, variando en función de la población (Tabla 2).

Los frutos que no son ni dispersados ni depredados caen al suelo debajo de la planta madre, en una cantidad que representó aproximadamente el $30 \%$ de la cosecha en tres poblaciones distintas del este de España (Verdú 1994). Experimentalmente se determinó que el $62 \%$ de los frutos caídos eran redispersados lejos de la planta madre. Teniendo en cuenta que el $13 \%$ de los frutos caídos contenían semillas viables (Verdú \& García-Fayos 1998a), el proceso de redispersión podría estar incrementando ligeramente el éxito reproductivo de las plantas.

\section{DEPREDACIÓN POSTDISPERSIÓN DE LAS SEMILLAS}

La probabilidad de que una semilla de P. lentiscus sea depredada va a depender, en gran medida, del sitio donde sea dispersada (Verdú \& García-Fayos 1996a). Los dispersantes suelen depositar las semillas preferentemente bajo árboles o arbustos que funcionan como perchas donde posarse y defecar o regurgitar la semillas (Debussche et al. 1985, Izhaki et al. 1991). Sin embargo, los depredadores no parece que rastreen las perchas en busca de semillas ya que las tasas de depredación bajo perchas no difieren de las encontradas en el suelo desnudo, ni en los matorrales ni en los campos de cultivo (Verdú \& García-Fayos 1996a). Tampoco se encuentra una mayor tasa de depredación bajo las hembras de P. lentiscus, como sugiere la hipótesis de Janzen-Connell (Janzen 1970, Connell 1971). Sin embargo, a distinta escala espacial, donde la vegetación se encuentra fragmentada en pequeñas áreas de bosque y matorral entremezclados con campos de cultivo en distintos estadios de abandono (Debussche \& Lepart 1992, Debussche \& Isenmann 1994), aparecen factores que afectan a las tasas de depredación. Así, dentro de un área de matorral, la probabilidad media de que una semilla sea depredada es del $63 \%$. Esta probabilidad incrementa con la relación perímetro/superficie del área de matorral porque grandes perímetros permiten la entrada de un mayor número de depredadores que viven en los ecotonos. Igualmente, las áreas de matorral con poca cobertura vegetal (que haría detectable visualmente a las semillas), o con mucha cobertura (que permitiría contener una alta densidad de semillas y/o refu-

\section{TABLA 2}

Porcentajes de dispersión de semillas de $P$. lentiscus en cinco poblaciones del Mediterráneo

Seed dispersal rates from five mediterranean populations of $P$. lentiscus

\begin{tabular}{lcl}
\hline Población & $\begin{array}{c}\text { Dispersión } \\
(\%)\end{array}$ & \\
\hline Beit-Jismal (Israel) & 91 & Izhaki \& Safriel (1985) \\
Doñana (España) & 83 & Jordano (1989) \\
Pujol (España) & 59 & Verdú \& García-Fayos (1995) \\
Porta-Coeli (España) & 49 & Verdú \& García-Fayos (1995) \\
Duna Punta (España) & 42 & Verdú \& García-Fayos (1995) \\
\hline
\end{tabular}


gios para los depredadores) tienen tasas de depredación más altas que las áreas con coberturas medias. Entre los campos de cultivo, la probabilidad de que una semilla sea depredada decrece al $27 \%$, siendo mayor en los campos que han sido abandonados, probablemente por poseer mayores densidades de semillas (Verdú \& García-Fayos 1996a). Además, cuanto más lejos del matorral esté el campo de cultivo, menor será la depredación (Verdú \& García-Fayos 1996a). En este sentido, el "anacronismo" que representa la dispersión lejana por medio de las aves es especialmente beneficioso para escapar a los depredadores de semilla en el paisaje mediterráneo que desde el final de las glaciaciones ha sido un mosaico muy dinámico de áreas abiertas con otras boscosas (Dupré 1988).

\section{GERMINACIÓN}

Otras características generalmente más conservativas, como son las asociadas a las semillas, muestran en esta especie patrones que podrían clasificarse como tropicales y que, por lo tanto, permanecerían inalterados desde su origen. Así, P. lentiscus cumple con las características que se han asociado a especies tropicales de germinación rápida después de las lluvias o muerte (Vázquez-Yáñes \& Orozco-Segovia 1993).

Las semillas de P. lentiscus carecen de mecanismos de latencia ya que no necesitan de ningún tipo de tratamiento para su germinación más que quitarles la pulpa, que es el papel que juegan las aves dispersantes (García-Fayos \& Verdú 1998). La germinación en condiciones de laboratorio es muy rápida, con un tiempo medio de germinación de 12,8 días (Piotto 1995) y un intervalo entre 4 y 28 días. En condiciones de invernadero y de campo se encontraron valores coherentes con estos experimentos, tardando las plántulas en emerger entre 23 y 100 días (García-Fayos \& Verdú 1998).

Las semillas que no germinan son incapaces de formar un banco permanente de semillas debido a que la viabilidad de las mismas decrece drásticamente después de un año (Troumbis $1991^{1}$, García-Fayos \& Verdú 1998).

Al contrario que las especies originadas en clima Mediterráneo, las semillas de P. lentiscus no son capaces de germinar tras un incendio ya

${ }^{1}$ TROUMBIS AY (1991) Zoochory and seed bank persistence in mediterranean type shrublands. Proceedings of the Sixth International Conference on Mediterranean Climate Ecosystems, Maléeme (Creta), Grecia, Septiembre 1991: 287-293. que mueren a temperaturas iguales o mayores a 70 ${ }^{\circ} \mathrm{C}$ (Salvador \& Lloret 1995, Verdú 2000). La estrategia seguida por esta especie tras los incendios es la de rebrotar vigorosamente a partir de yemas situadas en la base del tronco y de las reservas acumuladas en su extenso y ramificado sistema radicular (Naveh 1974).

Los requerimientos de germinación y establecimiento de plántulas son tales que el reclutamiento se podría producir casi anualmente con el régimen pluviométrico actual. Así pues, mantener la germinabilidad durante muchos años sólo serviría para evitar la muerte en los escasos años en que las condiciones favorables no se dieran. Sin embargo, además de la baja frecuencia con la que se producen estos períodos desfavorables (PérezCueva 1994, Jalut et al. 1997), las semillas remanentes en el suelo sufrirían tal depredación que comprometerían su permanencia en el banco de semillas. Por todo ello, esta estrategia tropical de germinación rápida tras las lluvias no ha debido verse penalizada en las condiciones climáticas mediterráneas actuales.

\section{ESTABLECIMIENTO DE PLÁNTULAS}

Las plántulas de $P$. lentiscus se establecen principalmente debajo de árboles o arbustos donde se posan las aves dispersantes y regurgitan o defecan las semillas (Debussche et al. 1982), fenómeno asociado a las áreas de matorral (García-Fayos \& Verdú 1998) y a los campos de cultivo (Verdú \& García-Fayos 1996b). Esto es debido a que las perchas, además de atraer aves dispersantes pero no depredadores, proporcionan un microclima adecuado para la germinación de semillas y emergencia de plántulas (Acherar et al. 1984, Verdú \& García-Fayos 1996a, 1996b, 1998b). Estas modificaciones microambientales positivas para el establecimiento de plántulas implica al menos una mayor disponibilidad hídrica y una menor compactación del suelo. Así pues, P. lentiscus puede verse beneficiado de este proceso de facilitación, muy común en zonas áridas (Bertness \& Callaway 1994), que mejora las duras condiciones mediterráneas actuales. Sin embargo, la protección de las perchas, al menos en las áreas de matorral, aunque mejore las condiciones de germinación y emergencia, no evita una alta tasa de mortalidad (fundamentalmente por sequía) de plántulas durante el primer año $(93 \%)$, similar a la que se produce en el suelo desnudo (98\%) (García-Fayos \& Verdú 1998).

Las plántulas establecidas en campos de cultivo, donde la competencia por recursos es baja, poseen un desarrollo mucho mayor que las esta- 
blecidas en matorrales. Así, mientras que en 5 años un individuo de $P$. lentiscus puede medir $165 \pm 46 \mathrm{~cm}$, en un matorral se han medido plántulas de 4 años con una altura de sólo $8 \pm 3 \mathrm{~cm}$ (Ne'eman \& Izhaki 1996, García-Fayos \& Verdú 1998). De nuevo, la capacidad de colonizar hábitats lejanos que le confiere la anacrónica dispersión endozoócora es vital para el éxito de esta especie.

\section{ESTRUCTURA DE LA POBLACIÓN}

Poco se sabe sobre la estructura de edades de las poblaciones de $P$. lentiscus ya que en esta planta es muy difícil determinar la edad. Esto es debido a que el crecimiento secundario anual puede detenerse según las condiciones ambientales y por lo tanto el número de anillos no corresponde al número de años de la planta (Fahn 1955, Liphschitz 1985, Abril \& Gracia 1992). Por otra parte, el tamaño de la planta puede ser poco indicativo de la edad de la misma ya que la planta es capaz de rebrotar tras perder la biomasa aérea (e.g., producto de incendios). Esta capacidad de rebrotar también representa un anacronismo, posiblemente desarrollado por sus ancestros ante la presión de herbivoría, aunque actualmente cumple la función de rebrote después del fuego (López-Soria \& Castell 1992, Lloret et al. 2000, Verdú 2000), que es otra perturbación frecuente en el mediterráneo (Naveh 1975). De esta manera, los individuos de $P$. lentiscus se autosuceden exitosamente después de un incendio.

En un campo abandonado de 11 años, Debussche et al. (1982) proporcionan una pirámide de edades de una población de P. lentiscus. En este campo de cultivo abandonado, los individuos de 3 años representan un $23 \%$ de la población, disminuyendo progresivamente el número de individuos de las clases de edades mayores. Las edades de 2 y 1 año representan el 17 y el $3 \%$ de la población, respectivamente, lo que supone que la tasa reclutamiento está ralentizándose. Estos resultados contrastan fuertemente con lo que ocurre en un área de matorral, donde el reclutamiento es muy escaso y además permanece constante entre años (García-Fayos \& Verdú 1998). En último término, con el paso del tiempo las estructuras poblacionales de edades de los matorrales y de los

TABLA 3

Porcentaje de hembras en distintas poblaciones Mediterráneas de P. lentiscus. Con un asterisco se señalan las proporciones que estadísticamente difieren del $50 \%$ (prueba de Chi-cuadrado o de G)

Sex ratio in different populations of P. lentiscus. An asterisk indicates significant statistical deviations from $1: 1$ sex ratio

\begin{tabular}{lll}
\hline Población & $\begin{array}{c}\text { Hembras } \\
(\%)\end{array}$ & \multicolumn{1}{c}{ Referencia } \\
\hline Convento (Portugal) & $23^{*}$ & Diaz-Barradas \& Correia (1999) \\
El Carmen (Portugal) & $24^{*}$ & Diaz-Barradas \& Correia (1999) \\
Top Ser. (Portugal) & $30^{*}$ & Diaz-Barradas \& Correia (1999) \\
Risco (Portugal) & $31^{*}$ & Diaz-Barradas \& Correia (1999) \\
Shemurat Zafririm (Israel) & $35^{*}$ & Rottenberg (1998) \\
Har Carmel (Israel) & 37 & Rottenberg (1998) \\
Mikhmoret (Israel) & $38^{*}$ & Rottenberg (1998) \\
Castelvolturno (Italy) & 40 & Martínez-Palle \& Aronne (2000) \\
Open oliv. (Portugal) & 41 & Diaz-Barradas \& Correia (1999) \\
Harifa (Israel) & 45 & Rottenberg (1998) \\
V. Rasca (Portugal) & 48 & Diaz-Barradas \& Correia (1999) \\
Doñana-1 (España) & 51 & Jordano (1988) \\
Doñana-2 (España) & 52 & Verdú \& García-Fayos (1998c) \\
Pego (España) & $55^{*}$ & Verdú \& García-Fayos (1998c) \\
PortaCoeli (España) & $56^{*}$ & Verdú \& García-Fayos (1998c) \\
Saler (España) & $61^{*}$ & Verdú \& García-Fayos (1998c) \\
Altura (España) & $61^{*}$ & Verdú \& García-Fayos (1998c) \\
Cabanes (España) & $63^{*}$ & Diaz Barradas \& Correia (1999) \\
Old oliv. (Portugal) & 63 & Verdú \& García-Fayos (1998c) \\
Dos Aguas (España) & $64^{*}$ & Hjalten et al. (1993) \\
La Carolina (España) & $64^{*}$ & Hjalten et al. (1993) \\
El Rocío (España) & $69^{*}$ & \\
\hline
\end{tabular}


campos de cultivo colonizados por P. lentiscus convergerían. Así, a partir de la dinámica de regeneración, sería esperable que la estructura de edades en matorrales estables fuera la del predominio de edades viejas y ausencia casi total de edades jóvenes, como ocurre por ejemplo en Quercus ilex (Espelta et al. 1995).

La estructura de sexos ha sido explorada en muchas poblaciones de $P$. lentiscus encontrándose proporciones sexuales tanto sesgadas hacia hembras como a machos y también en equilibrio (Tabla 3). El único patrón que se ha encontrado para explicar la proporción sexual es que las zonas más perturbadas suelen tener mayor proporción de hembras que las no perturbadas (Verdú \& García-Fayos, 1998c, Díaz-Barradas \& Correia 1999). Este hecho puede explicarse, en parte, debido a que los machos poseen una mayor capacidad competitiva bajo condiciones de recurso limitado, como es el agua (Correia \& DíazBarradas 2000). Por otra parte, es probable que la explotación humana de $P$. lentiscus haya sido diferencial, conservando en mayor proporción las hembras para explotar el aceite de sus frutos y atraer aves para cazar y por otro lado cortando los machos para leña y carbón (Verdú \& GarcíaFayos 1998c). En definitiva, la estructura sexual varía ampliamente entre sus poblaciones como un efecto de la perturbación del hábitat, que es algo común en la cuenca mediterránea.

\section{CONCLUSIONES}

El ciclo reproductivo de $P$. lentiscus está constituido por una amalgama de caracteres tropicales que le sirven no sólo para sobrevivir en el matorral mediterráneo, sino para colonizar los nuevos hábitats surgidos de la destrucción de éste. Este hecho se vería corroborado por el registro palinológico, que muestra una expansión de esta especie desde la aparición del hombre (Pons 1981).

\section{AGRADECIMIENTOS}

Especial agradecimiento merece Anna Traveset por su invitación al Taller de la Red Iberoamericana de Ecosistemas Mediterráneos celebrado en Mallorca del que surgió este trabajo. Durante la redacción de este trabajo, M.V. disfrutó de un contrato de Reincorporación de Doctores y Tecnólogos del Ministerio de Educación y Ciencia. El proyecto FEDER 1FD97-0551 financió los gastos del presente trabajo.

\section{LITERATURA CITADA}

ABELTINO PM (1996) Studio del germoplasma di Pistacia lentiscus L. per la selezione di ecotipi ad alta efficienza riproduttiva. Tesis de licenciatura, Facolta d’Agraria, Universitat'degli Studi di Sassari, Sassari, Italia. 103 pp.

ABRILE M \& C GRACIA (1989) Crecimiento de los rebrotes de Pistacia lentiscus y Quercus coccifera después de un incendio. Options Méditerranéennes, Série Séminaires 3: 101-106.

ACHERAR M, J LEPART \& M DEBUSSCHE (1984) La colonisation des frisches par le pin d'Alep (Pinus halepensis) en Languedoc mediterraneen. Acta Oecologica 5: 179-189.

ARISTA M, J ROSSO \& S TALAVERA (1990) Caracterización de Pistacia x saportae Burnat, P.lentiscus L. y P.terebinthus L. en la Serranía de Grazalema. Lagascalia 16: 311-322.

AXELROD DI (1973) History of the mediterranean ecosystem in California. En: Di Castri F \& HA Mooney (eds) Mediterranean-type ecosystems: 225-277. Springer-Verlag, Berlin, Alemania.

AXELROD DI (1975) Evolution and biogeography of Madrean-Tethyan sclerophyll vegetation. Annals of the Missouri Botanical Garden 62: 280-334.

BALLOUCHE A (1986) Paleoenvironnements de l'homme fossile holocene au Maroc: Apports de la palynologie. Tesis de Doctorado (Ph.D.), Université de Bordeaux, Bordeaux, Francia. 141 pp.

BERTNESS MD \& R CALlAWAY (1994) Positive interactions in communities. Trends in Ecology and Evolution 9: 191-193.

CONNELL JH (1971) On the role of natural enemies in preventing competitive exclusion in some marine animals and in rain forest trees. En: den Boer PJ \& GR Gradwell (eds) Dynamics of populations: 298-312. Centre for Agricultural Publishing and Documentation, Wageningen, The Netherlands.

CORREIA OA, AC MARTINS \& FM CATARINO (1992) Comparative phenology and seasonal foliar nitrogen variation in mediterranean species of Portugal. Ecologia Mediterranea 18: 7-18.

CORREIA OA \& MC DÍAZ-BARRADAS (2000) Ecophysiological differences between male and female plants of Pistacia lentiscus. Plant Ecology 149: 131-142.

DE LILLIS M \& A FONTANELLA (1992) Comparative phenology and growth in different species of the Mediterranean maquis. Vegetatio 99/100: 83-96.

DEBUSSCHE M \& J LEPART (1992) Establishment of woody plants in mediterranean old fields: opportunity in space and time. Landscape Ecology 6: 133-145.

DEBUSSCHE M \& P ISENMANN (1994) Bird-dispersed seed rain and seedling establishment in patchy Mediterranean vegetstion. Oikos 69: 414-420.

DEBUSSCHE M, J ESCARRÉ \& J LEPART (1982) Ornithochory and plant succession in Mediterranean abandoned orchards. Vegetatio 48: 255-266.

DEBUSSCHE M, J LEPART \& J MOLINA (1985) La dissémination des plantes à fruits charnus par les oiseaux: rôle de la structure de la végétation et impact sur la succession en région méditerranéenne. Acta Oecologica 6: 65-80. 
DEBUSSCHE M, J CORTÉZ \& I RIMBAULT (1987) Variation in fleshy fruit composition in the Mediterranean region: the importance of ripening season, life-form, fruit type and geographical distribution. Oikos 49: 244-252

DE LILLIS M \& A FONTANELLA (1992) Comparative phenology and growth in different species of the Mediterranean maquis. Vegetatio 99/100: 83-96.

DÍAZ-BARRADAS MC \& OA CORREIA (1999) Sexual dimorphism, sex ratio nad spatial distribution of male and female shrubs in the dioecious species Pistacia lentiscus L. Folia Geobotanica 34: 163-174.

DUPRÉ M (1988) Palinología y paleoambiente: nuevos datos españoles, referencias. Servei d’Investigació Prehistórica, Valencia, España. 160 pp.

ESPELTA JM, M RIBA \& J RETANA (1995) Patterns of seedling recruitment in west-Mediterranean Quercus ilex forests influenced by canopy development. Journal of Vegetation Science 6: 465-472.

FAHN A (1955) The development of the growth ring in wood of Quercus infectoria and Pistacia lentiscus in the hill region of Israel. Tropical Woods 101: 52-60.

GARCÍA-FAYOS P \& M VERDÚ (1998) Soil seed bank, factors controlling germination and establishment of a Mediterranean shrub: Pistacia lentiscus L. Acta Oecologica 19: 357-366.

GRUNDWAG M (1975) Seed set in some Pistacia L. (Anacardiaceae) species after inter- and intraspecific pollination. Israel Journal of Botany 24: 205-211.

GRUNDWAG M (1976) Embriology and fruit development in four species of Pistacia L. (Anacardiaceae). Botanical Journal of the Linnean Society 73: 355370.

HERRERA CM (1982a) Vertebrate-dispersed plants of the Iberian peninsula: a study of fruit characteristics. Ecological Monographs 57: 305-331

HERRERA CM (1982b) Seasonal variation in the quality of fruits and diffuse coevolution between plants and avian dispersers. Ecology 63: 773-785.

HERRERA CM (1984a) A study of avian frugivores, birddispersed plants and their interaction in Mediterranean scrublands. Ecological Monographs 54: 1-23.

HERRERA CM (1984b) Tipos morfológicos y funcionales en plantas del matorral mediterraneo del sur de España. Studia Oecologica 5: 7-34.

HERRERA CM (1988) Variaciones anuales en las poblaciones de pájaros frugívoros y su relación con la abundancia de frutos. Ardeola 35: 135-142.

HERRERA CM (1992) Historical effects and sorting processes as explanations for contemporary ecological patterns: character syndromes in mediterranean woody plants. American Naturalist 140: 421-446.

HERRERA J (1987) Flower and fruit biology in southern Spanish Mediterranean shrublands. Annals of the Missouri Botanical Garden 74: 69-78.

HJÄLTÉN J, M ASTRÖM, E ABERG \& K DANELL (1993) Biased sex ratio in spanish populations of Pistacia lentiscus (Anacardiaceae): the possible role of herbivory. Anales Jardín Botánico de Madrid 51: 49-53.

IZHAKI I \& UN SAFRIEL (1985) Why do fleshy-fruit plants of the mediterranean scrub intercept fall -but not spring- passage of seed-dispersing migratory birds?. Oecologia 67: 40-43.
IZHAKI I, PB WALTON \& UN SAFRIEL (1991) Seed shadows generated by frugivorous birds in an eastern mediterranean scrub. Journal of Ecology 79:575-590.

JALUT G, A ESTEBAN-AMAT, S RIERA, I MORA, M FONTUGNE, $\mathrm{R}$ MOOK, $\mathrm{L}$ BONNET \& $\mathrm{T}$ GAUQUELIN (1997) Holocene climatic changes in the western Mediterranean: instalation of the Mediterranean climate. Earth and Planetary Sciences 325: 327-334.

JANZEN DH (1970) Herbivores and the number of tree species in tropical forests. American Naturalist 104:501-528.

JORDANO P (1984) Relaciones entre plantas y aves frugívoras en el matorral mediterráneo del área de Doñana. Tesis Doctoral, Universidad de Sevilla, Sevilla, España. 305 pp.

JORDANO P (1988) Polinización y variabilidad de la producción de semillas en Pistacia lentiscus L. (Anacardiaceae). Anales Jardín Botánico de Madrid 45: 213-231.

JORDANO P (1989) Pre-dispersal biology of Pistacia lentiscus (Anacardiaceae): cummulative effects on seed removal by birds. Oikos 55: 375-386.

JORDANO P (1990) Utilización de los frutos de Pistacia lentiscus (Anacardiaceae) por el verderón común (Carduelis chloris). En: Arias de Reyna L, P Recuerda \& T Redondo (eds) Actas Primer Congreso Nacional de Etología: 145-153. Cajasur, Córdoba, España.

LIPHSCHITZ N, S LEV-YADUN \& Y WAISEL (1985) The annual rhythm of the lateral meristems (cambium and phellogen) in Pistacia lentiscus L. IAWA Bulletin 6: 239-244.

LLORET F, M VERDÚ, N FLORES-HERNÁNDEZ \& A VALIENTE-BANUET (1999) Fire and resprouting in Mediterranean ecosystems: insights from an external biogeographical region, the Mexican shrubland. American Journal of Botany 86: 1655-1661.

MARTÍNEZ-PALLE E \& G ARONNE (2000) Reproductive cycle of Pistacia lentiscus (Anacardiaceae) in southern Italy. Plant Biosystems 134: 365-371.

NAVEH Z (1974) Effects of fire in the mediterranean region. En: Kozlowsky TT \& CE Ahlgren (eds) Fire and ecosystems: 401-434. Academic Press, New York, New York.

NE'EMAN G \& I IZHAKI (1996) Colonization in an abandoned east-mediterranean vineyard. Journal of Vegetation Science 7: 465-472.

PALAMAREV E (1989) Paleobotanical evidences of the Tertiary history and origin of the Mediterranean sclerophyll dendroflora. Plant Systematics and Evolution 162: 93-107.

PARFITT DE \& ML BADENES (1997) Phylogeny of the genus Pistacia as determined from analysis of the chloroplast genome. Proceedings of the National Academy of Sciences USA 94: 7987-7992

PÉREZ-CUEVA AJ (1994) Atlas Climàtic de la Comunitat Valenciana. Consellería d'Obres Públiques, Urbanisme i Transports, Valencia, España. 205 pp.

PIOTTO B (1995) Influence of scarification and prechilling on the germination of seeds of Pistacia lentiscus. Seed Science and Technology 2: 659-663. 
PONS A (1981) The history of the mediterranean shrublands. En Di Castri F, DW Goodall \& RL Spetch (eds) Mediterranean-type shrublands: 131-138. Elseviere, Amsterdam, The Netherlands.

QUEZEL P (1981) Floristic composition and phytosociological structure of sclerophyllous matorral around the mediterranean. En: Di Castri F, DW Goodall \& RL Spetch (eds) Mediterranean-type shrublands: 107-121. Elseviere, Amsterdam, The Netherlands.

RAVEN PH (1973) Plant biogeography: introduction. En: Di Castri F \& HA Mooney (eds) Mediterranean-type ecosystems: 211-212. Springer-Verlag, Berlin, Alemania.

ROTTENBERG A (1998) Sex ratio and gender stability in the dioecious plants of Israel. Botanical Journal of the Linnean Society 128: 137-148.

SALVADOR R \& F LLORET (1995) Germinación en el laboratorio de varias especies arbustivas mediterráneas: efecto de la temperatura. Orsis 10: 25-34.

SCARAMUZZI F (1957) Il ciclo riproduttivo di Pistacia lentiscus L. Nuovo Giornale Botanico Italiano 1-2: 198-213.

SUC JP (1984) Origin and evolution of the mediterranean vegetation and climate in Europe. Nature 307: 429432.

TRAVESET A (1993) Deceptive fruits reduce insect seed predation in Pistacia terebinthus L. Evolutionary Ecology 7: 357-361.

VÁZQUEZ-YÁÑES C \& A OROZCO-SEGOVIA (1993) Patterns of seed longevity and germination in the tropical rainforest. Annual Review of Ecology and Systematics 24: 69-87.

VERDÚ M (1994) Regeneración y colonización de Pistacia lentiscus: procesos y patrones. Tesis Doctoral, Facultad de Biología, Universidad de Valencia, Valencia, España. 173 pp.
VERDÚ M \& P GARCÍA-FAYOS (1994) Correlations between the abundances of fruits and frugivorous birds: the effect of temporal autocorrelation. Acta Oecologica 15: 791-796.

VERDÚ M \& P GARCÍA-FAYOS (1995) Dispersión y predación predispersiva de semillas en Pistacia lentiscus. Studia Oecologica 12: 169-178.

VERDÚ M \& P GARCÍA-FAYOS (1996a) Postdispersal seed predation in a mediterranean patchy landscape. Acta Oecologica 17: 379-391.

VERDÚ M \& P GARCÍA-FAYOS (1996b) Nucleation processes in a mediterranean bird-dispersed plant. Functional Ecology 10: 275-280.

VERDÚ M \& P GARCÍA-FAYOS (1998a) Ecological causes, function, and evolution of abortion and parthenocarpy in Pistacia lentiscus (Anacardiaceae). Canadian Journal of Botany 76: 134-141.

VERDÚ M \& P GARCÍA-FAYOS (1998b) Old-field colonization by Daphne gnidium: seedling distribution and spatial dependence at different scales. Journal of Vegetation Science 9: 713-718.

VERDÚ M \& P GARCÍA-FAYOS (1998c) Female biased sex ratios in Pistacia lentiscus L. (Anacardiaceae). Plant Ecology 135: 95-101.

VERDÚ M \& P GARCÍA-FAYOS (2001) The effect of deceptive fruits on predispersal seed predation by birds in Pistacia lentiscus. Plant Ecology 156: 245248.

VERDÚ M (2000) Ecological and evolutionary differences between mediterranean seeders and resprouters. Journal of Vegetation Science 11: 265-268.

ZOHARY M (1952) A monographical study of the genus Pistacia. Palestine Journal of Botany 4: 187-228. 